\title{
A comparison of neutron star blackbody luminosities in LMXB with the theory of accretion flow spreading on the stellar surface
}

\author{
M. J. Church ${ }^{1,2}$, N. A. Inogamov ${ }^{3}$, and M. Bałucińska-Church ${ }^{1,2}$ \\ 1 School of Physics and Astronomy, University of Birmingham, Birmingham B15 2TT, UK \\ e-mail:mjc@star.sr.bham.ac.uk;mbc@star.sr.bham.ac.uk \\ 2 Astronomical Observatory, Jagiellonian University, ul. Orla 171, 30-244 Cracow, Poland \\ 3 Landau Institute for Theoretical Physics, Russian Academy of Science, Kosygin Street 2, V-334, 117940 Moscow, \\ Russian Federation \\ e-mail: nail@landau.ac.ru
}

Received 26 June 2001 / Accepted 11 April 2002

\begin{abstract}
We present a comparison of the results of the ASCA survey of LMXB with the Inogamov \& Sunyaev theory of accretion flow spreading on the surface of neutron stars. The ASCA survey of LMXB of Church \& Bałucińska-Church (2001) revealed a systematic variation of the luminosity of blackbody emission from the neutron star spanning 3 decades in total X-ray luminosity suggesting that the level of blackbody emission is controlled by the physics of the inner disk/stellar interface, which we can hope to understand. Two types of explanation exist: firstly that there is radial flow between the inner disk and star at all vertical positions above the orbital plane so that the height of the disk directly determines the area of star emitting. Secondly, the height of the emitting region on the star is not directly related to the disk properties but depends on the mass accretion rate as suggested by Inogamov \& Sunyaev (1999) in their theory of accretion flow spreading on the stellar surface. We find that the survey results for the emitting area agree with this theory at the lowest luminosities. However, for higher luminosities, the blackbody emission is stronger than predicted by spreading theory suggesting that the emitting area is controlled by radial flow between disk and star.
\end{abstract}

Key words. X rays: stars - stars: neutron - binaries: close - accretion, accretion disks

\section{Introduction}

The nature of the X-ray emission in Low Mass X-ray Binaries (LMXB) has for many years been controversial. It is clear that the accretion flow deposits its energy partly in the accretion disk resulting in heating and X-ray emission, and partly in a boundary layer at the surface of the star or on the stellar surface itself, but the nature of the emission has not been agreed. Firstly, there has been the question of how many continuum components there are. The spectra of the less bright LMXB are clearly dominated by Comptonization which led to the use of the Generalized Thermal model $E^{-\Gamma} \cdot \mathrm{e}^{-E / E_{\mathrm{CO}}}$ in the fitting of Exosat spectra (White et al. 1988). Only in the case of brighter sources did spectral fitting demand that a blackbody component was added to this. Mitsuda et al. (1989) proposed a model consisting of multi-colour disk (MCD) blackbody emission from the inner accretion disk plus blackbody emission from the neutron star, Comptonized in a region local to the star, and this two-component model is able to fit many

Send offprint requests to: M. J. Church, e-mail:mjc@star.sr.bham.ac.uk sources well. Secondly, there is the identification of the blackbody component in LMXB spectra. Opinion has been divided between location on the surface or boundary layer at the star, or in the inner accretion disk, and this controversy continues to recent times. There are also physical differences between models involving $\mathrm{X}$-ray emission in the boundary layer and those involving the stellar surface (see below). In the former case, it is assumed that the angular momentum in the inner disk adjusts to that of the star in a boundary layer in the disk which becomes the source of X-rays. Thirdly, the location, geometry and size of the Comptonizing region have been controversial. Many theoreticians have favoured Comptonization taking place in an inner, hot region close to the neutron star.

However, the dipping class of LMXB provides answers to these problems although this has not been widely appreciated. This group of $\sim 10$ sources exhibit decreases in X-ray intensity at the orbital period due to absorption in the bulge in the outer disk where the accretion flow from the companion star impacts. Emission models are more strongly constrained in these sources, as models have to fit not only the spectrum of the quiescent source as in other types of LMXB, but also several levels 
of dipping. Spectral evolution is not simple, and cannot be fitted by absorbed one-component models. It has been shown by analysis of data from many observations with Exosat, ASCA, BeppoSAX and Rossi-XTE that these sources are well-described by a model consisting of point-like blackbody emission plus Comptonized emission from an extended accretion disk corona (ADC) (e.g. Church et al. 1997, 1998; Bałucińska-Church et al. 1999, 2000, 2001; Smale et al. 2001). The blackbody is absorbed rapidly in dipping showing that it is point-like, and has temperatures between 0.9 and $\sim 2 \mathrm{keV}$. The Comptonized emission is shown by spectral analysis to be removed gradually, and the complex spectral evolution is well-described by progressive covering of this component in dipping by the absorber, the covering fraction rising smoothly in many cases from zero to unity. This, of course, requires the Comptonizing region to be extended allowing the size to be estimated. Moreover, measurement of dip ingress times directly provides the size of the region, and values of the radius of this region for several sources typically of $50000 \mathrm{~km}$ have been determined (Church 2001). It is clear that a very extended Comptonizing region with high electron temperature can only be identified with an ADC. The dipping sources also indicate that this must be a thin corona, since if an ADC of this large radius was spherical, it is unlikely that the absorber on the outer disk would be able to overlap the source region completely to produce the $100 \%$ deep dipping observed in many sources (Smale et al. 2001). It is likely that the ADC in all dipping LMXB is very extended, which can probably be related to the strong effects of the neutron star forming the ADC by evaporation of the accretion disk. Moreover, it is not expected that the dipping sources are atypical, and so an extended ADC is expected in all LMXB. The large measured size of the ADC has several significant consequences as discussed below.

Firstly, these measurements of the ADC radius $r_{\mathrm{ADC}}$ allow us to rule out Comptonization models that involve a localized region in the neighbourhood of the neutron star. Secondly, the ADC size measurements have an important consequence for blackbody emission observed from LMXB. It is expected that a substantial fraction of the total available energy will be dissipated initially in the accretion disk, implying a large disk blackbody component. However, because the ADC is very extended, all of the hot inner regions of the disk will be covered by ADC, and consequently it is expected, given the high optical depth of the ADC (Church 2001), that all disk blackbody emission will become Comptonized in the ADC so that no blackbody emission from the disk itself should be visible. It is also clear on the basis of the thin, flat disk and thin, flat ADC geometry, that thermal emission from the disk must be the major source of seed photons for Comptonization in the ADC. Thus, blackbody emission clearly identified in the spectra of many LMXB (e.g. Church \& Bałucinska-Church 2001, see below) must be identified with the neutron star/boundary layer without any contribution from the disk being expected. This contrasts with a recent tendency to fit a disk blackbody plus comptt Comptonization model routinely to BeppoSAX data (e.g. Guainazzi et al. 1998; Sidoli et al. 2001; Oosterbroek et al. 2001). This is thus inconsistent with the implications of the large measured sizes of Accretion Disk Coronae.
The large ADC size is also relevant to the comptt Comptonization model used in the above fitting. It has been claimed that this is preferable at low energies $(<1 \mathrm{keV})$ to a cut-off power law representation of Comptonization as the latter implies that the spectral flux density continues to increase with decreasing photon energy, even for very low energies below $0.1 \mathrm{keV}$. Clearly, at very low energies the flux must decrease because of the lack of seed photons. The comptt model due to Titarchuk (1994) calculates a Comptonized spectrum assuming a sea of soft thermal photons and so avoids an increase of flux at the lowest energies. It uses the Wien approximation to the seed photon spectrum, valid for $h v \gg k T$. However, usage of this model in which values of $k T$ from spectral fitting as high as $1 \mathrm{keV}$ and $2 \mathrm{keV}$ are derived (e.g. Guainazzi et al. 1998) will contravene the assumptions of the model, and will overestimate the seed photon spectrum, and thus the Comptonized spectrum by as much as $100 \%$ at $1 \mathrm{keV}$. Thus a model with $k T=2 \mathrm{keV}$ will only be valid above $10 \mathrm{keV}$. Regarding the availability of seed photons, we can calculate whether there is any shortage of seed photons below $1 \mathrm{keV}$ as follows. The large size of the ADC referred to above ensures that seed photons produced in the accretion disk out to a radius of $\sim 50000 \mathrm{~km}$ will be Comptonized as the disk is covered by ADC out to this radius. We have calculated the multi-colour disk blackbody spectrum of this part of the disk assuming a thin disk temperature profile $T(r)$ by integration in radius (for appropriate values of mass accretion rate). It is clearly dominated by the soft photons from outer radii. For a luminosity of $10^{36} \mathrm{erg} \mathrm{s}^{-1}$, the photon spectral flux density forms a broad peak between $0.001-0.01 \mathrm{keV}$, and for a luminosity of $10^{38} \mathrm{erg} \mathrm{s}^{-1}$, the flux peaks between $0.01-0.1 \mathrm{keV}$, showing that there is no deficiency of low energy photons. Thus, the cut-off power law is a perfectly good description of the Comptonized spectrum to energies at least as low as $0.1 \mathrm{keV}$, whereas application of the comptt model without restricting $k T$ for the seed photons to suitably low values will be invalid.

Finally, the extended size of the ADC has implications for reflection in LMXB. Because all of the inner disk is covered by $\mathrm{ADC}$, illumination of the disk by the neutron star will not be possible, and "hot" reflection of this source from the ADC will take place. This will not however, have the absorption features of a reflection component from the accretion disk. Moreover, although the ADC itself can illuminate the disk and lead to reflection, the high values of optical depth obtained for the ADC (Church 2001) suggest that this component will be reprocessed in the ADC and so not observed. This may explain the lack of detections of reflected components in LMXB. In a black hole binary such as Cyg X-1, the ADC radius is much smaller (Church 2001), and so a reflection component can be expected, as observed.

\subsection{The ASCA survey}

The above two-component model used to explain dipping LMXB spectra has been applied recently to a survey of all classes of LMXB, including the Atoll and Z-track sources (Church \& Bałucinska-Church 2001), and found to fit all 


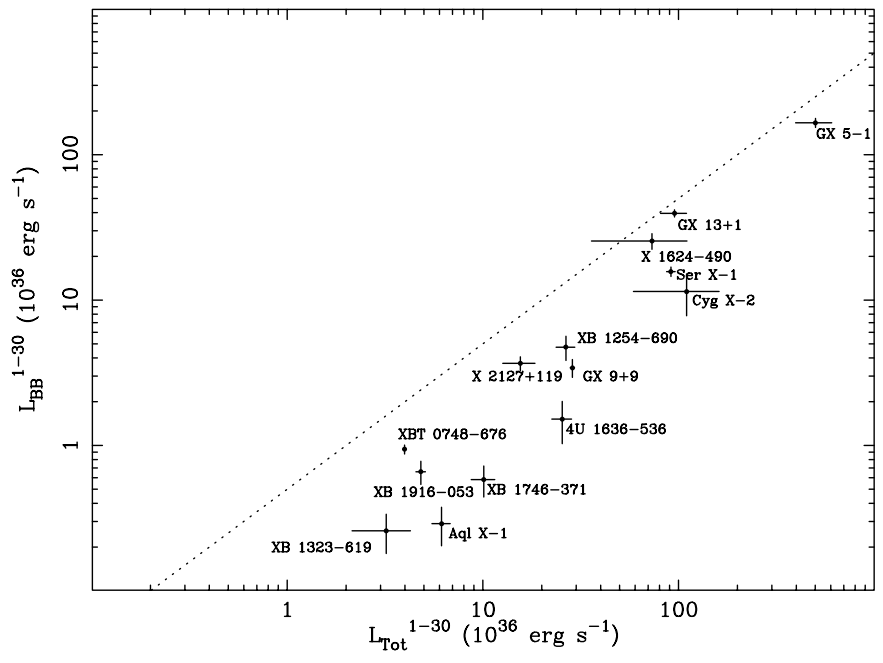

Fig. 1. Variation of blackbody luminosity in the band $1-30 \mathrm{keV}$ with total luminosity in the same band from the ASCA survey of LMXB of Church \& Bałucińska-Church (2001).

sources well. As part of this survey, it was shown that the blackbody emission cannot originate in the accretion disk, since fitting a two-component model consisting of multi-colour disk blackbody plus a Comptonization term gave in many cases a value for the inner disk radius $r_{\mathrm{i}}$ more than 10 times smaller than the radius of the neutron star assumed to be $\sim 10 \mathrm{~km}$. Thus the blackbody emission has to be from the neutron star and these results support the idea that disk blackbody emission is fully Comptonized in the ADC. The main result of the survey was the systematic behaviour that it revealed in the neutron star blackbody emission as reproduced in Fig. 1. In brighter sources, the 1-30 keV blackbody luminosity $L_{\mathrm{BB}}$ approached $50 \%$ of the total luminosity (the dotted line) as expected in the Newtonian approximation. In fainter sources, $L_{\mathrm{BB}}$ fell to much smaller fractions of the total. It was demonstrated that, as the total luminosity increased, the blackbody emitting area increased by a factor of 5400 across the sample, whereas the blackbody temperature $k T_{\mathrm{BB}}$ changed (in fact, decreased) by $40 \%$ (i.e. a factor of 0.60 ), corresponding to a change by a factor of 8 in $T^{4}$. Thus, the area is the most important factor in determining the level of blackbody emission.

\subsection{Possible modification of the blackbody}

The survey assumed that there was simple blackbody emission from the surface of the neutron star, and we next discuss this assumption. Firstly, modification of the blackbody was proposed by Rutledge et al. (1999) for transiently-accreting LMXB in quiescence, having $k T$ typically $0.2 \mathrm{keV}$ and luminosity $L \sim$ $10^{33} \mathrm{erg} \mathrm{s}^{-1}$. The modification arises from higher energy photons escaping from greater depths in the atmosphere. This is clearly inapplicable to LMXB not in quiescence emitting at luminosities of $10^{36}-10^{38} \mathrm{erg} \mathrm{s}^{-1}$ (Rutledge priv comm).

Secondly, although in the study of X-ray bursts there is observational evidence that there is sometimes modification of the blackbody by electron scattering in the neutron star atmosphere during bursts, there is no such evidence in the case of non-burst emission. Fitting a simple blackbody in a case where modification did take place would lead to errors in $k T$ and the emitting area. There are two pieces of evidence for modification in bursts (see the detailed discussion in Bałucińska-Church et al. 2001). Firstly, values of $k T$ of $\sim 3 \mathrm{keV}$ have been obtained for a fraction of bursts from fitting a simple blackbody to the peak, which implies super-Eddington emission. Secondly, an increase in blackbody radius $R_{\mathrm{BB}}$ while the blackbody temperature decreased during burst decay was found in the source XB 1636-536 (Inoue et al. 1984; Sztajno et al. 1985 ). The increase of $R_{\mathrm{BB}}$ was thought to be unreal and a consequence of fitting a simple blackbody when the emission was modified blackbody. However, whether this effect is observed depends on whether spectral fitting allows for blackbody emission by the non-bursting source (Sztajno et al. 1986), and so is not strong evidence for modification. More recently, Kuulkers et al. (2002) have presented evidence that during the cooling phase of burst decay in GX 17+2, the blackbody is not modified.

In fact, whether modification takes place depends critically on the electron density $n_{\mathrm{e}}$ which determines whether absorption processes or electron scattering dominate the opacity. Various theoretical descriptions of the neutron star atmosphere in bursting have been given, by Fujimoto et al. (1981), Paczyński (1983), London et al. (1984, 1986), Ebisuzaki et al. (1984), Ebisuzaki (1987) and Madej (1991). However, there are very large disparities between the electron density values in these works, $n_{\mathrm{e}}$ varying between $10^{24}-10^{32} \mathrm{~cm}^{-3}$. While an atmosphere with $n_{\mathrm{e}}=10^{24} \mathrm{~cm}^{-3}$ is clearly dominated by electron scattering, a higher density atmosphere is not. Thus the theoretical picture is unclear and we have to rely on observational evidence that some bursts only have a modified spectrum. For these, the effect of modification on measured values of $k T$ depends on the ratio of colour temperature to effective temperature $T_{\text {col }} / T_{\text {eff }}$ for which recent theoretical values are low, i.e. 1.1 in the work of Madej \& Różańska (2000) (Czerny, priv comm), compared with earlier values which were higher. Values can be derived using the London et al. model of $\sim 1.6$ for $k T_{\text {eff }}=2 \mathrm{keV}$ appropriate to a burst peak, $\sim 1.3$ for $1.5 \mathrm{keV}$, and $\sim 1.1$ for $1 \mathrm{keV}$ (assuming hydrogen composition). Thus even these factors imply relatively small corrections for non-burst emission. The effect on emitting area depends on this ratio squared, so in terms of recent theory can reach only $20 \%$. In non-burst emission, there is actually no evidence for modification at all and so it would be inappropriate to apply a correction factor. On the contrary, the ASCA survey results in which the blackbody radius approaches $10 \mathrm{~km}$ in bright sources, i.e. consistent with the neutron star radius, provide evidence that there is no large modification. Thus it is unlikely that the results of the present paper would be affected.

In Fig. 2, the $A S C A$ survey results are replotted in the form of half-height $h$ of the emitting region on the neutron star assumed to be an equatorial strip, more specifically, a sphere intersected by two parallel planes, having emitting area $4 \pi R h$ where $R$ is the radius of the neutron star. $h$ varied between $20 \mathrm{~m}$ (at the lowest luminosities) and the full radius of the star $\sim 10 \mathrm{~km}$. These heights are (for almost all of the data) 


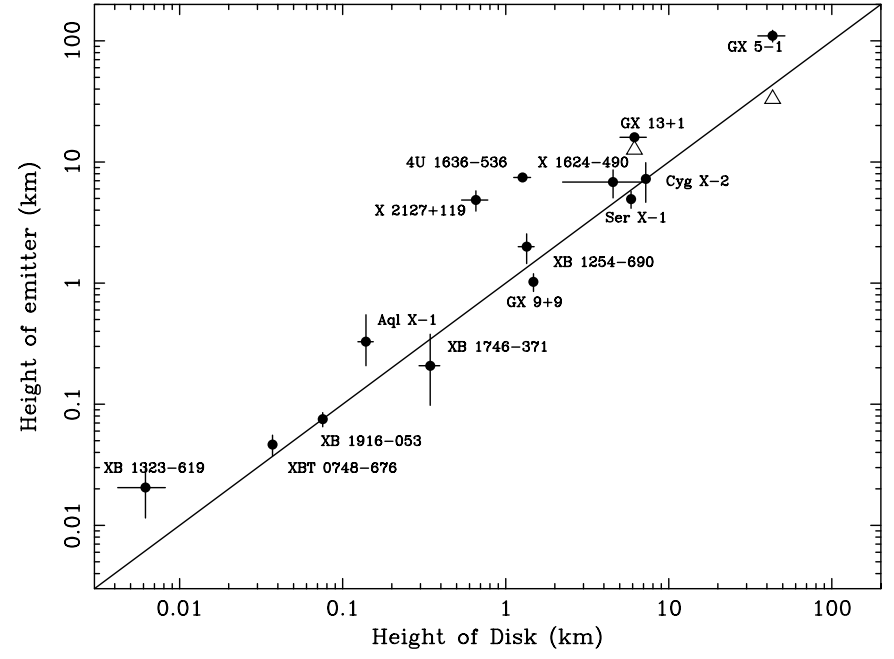

Fig. 2. Variation of the height $h$ of the blackbody emission region on the surface of the neutron star with the height of the inner, radiativelysupported accretion disk calculated at the radial position where $p_{\mathrm{r}}=10 p_{\mathrm{g}}$.

substantially larger than the half-height of the inner accretion disk calculated using thin disk theory (Shakura \& Sunyaev 1976) at some position close to the surface of the neutron star (e.g. 1.001R). This thin disk half-height is $\sim 20 \mathrm{~m}$ for a luminosity of $10^{36} \mathrm{erg} \mathrm{s}^{-1}$ and $\sim 40 \mathrm{~m}$ for $10^{38} \mathrm{erg} \mathrm{s}^{-1}$. However, the inner disk is expected to be radiatively supported over a wide range of luminosities $>3 \times 10^{36} \mathrm{erg} \mathrm{s}^{-1}$ and consequently has much larger vertical extent. The radiatively-supported disk merges at some radius with the thin disk and the half-height $H\left(r_{10}\right)$ was calculated at the radius at which the radiation pressure was 10 times the gas pressure (Czerny \& Elvis 1987). Remarkably, there was good agreement between $h$ and $H\left(r_{10}\right)$ for the majority of the survey sources, the agreement spanning 3 decades in each quantity as shown in Fig. 2 (Fig. 3, Church $\&$ Bałucińska-Church 2001). This agreement suggests that the properties of the inner disk determine in some way the blackbody emitting area. In the case of the bright source GX 5-1, the agreement is poor with $h=110 \mathrm{~km}$ and $H\left(r_{10}\right)=43 \mathrm{~km}$. The blackbody radius $R_{\mathrm{BB}}$ is $33 \mathrm{~km}$ showing that the data cannot be explained by emission from the neutron star alone, suggesting that the emitting region has expanded to a spherical cloud centred on the star. However, there is good agreement between the height of the cloud at $33 \mathrm{~km}\left(=R_{\mathrm{BB}}\right)$ plotted as a triangle in Fig. 2 and $H\left(r_{10}\right)$. A similar point is plotted for the blackbody radius of the source $\mathrm{GX} 13+1$. There is thus some evidence that, for these points also, $H$ determines $h$. In the present paper, we compare the survey results with possible mechanisms leading to the agreement of $h=H$, specifically i) radial flow between the accretion disk and neutron star, and ii) accretion flow spreading on the surface of the neutron star (Inogamov \& Sunyaev 1999).

The agreement between $h$ and $H$ suggests that the properties of the inner disk determine the blackbody emitting area in some way. However, there are at least two possible explanations of the agreement. Firstly, there may be radial flow across the gap between the inner disk and the star at all vertical positions above the orbital plane so that the disk height approximately sets the height of the emitting area on the star. This requires there to be a stable, radiatively-supported inner accretion disk, although it has been suggested that the radiative disk is subject to instabilities. These may not however, disrupt the disk in a major way as their characteristic timescale is short (see Sect. 4). Secondly, there may be spreading of the accretion flow on the neutron star surface as proposed by Inogamov \& Sunyaev (1999). In this case, accretion flow between the disk and the star will be essentially in the orbital plane. In this mechanism discussed below, the accretion flow spreads vertically over the neutron star, the height reached depending on the mass accretion rate and thus on the total luminosity. The emitting area depends on $L$, and the quantity $H$ is simply a measure of $L$; thus the agreement of $h$ with $H$ represents the variation of emitting area with luminosity (and does not result from a direct mapping of the disk height onto the star). We next compare the survey results with this theory.

\section{Accretion flow on the surface of the neutron star}

In the often accepted standard theory of accretion flow interaction with neutron stars, it is assumed that the adjustment in angular velocity between the accreting material with high Keplerian velocity and the slower rotation of the star takes place in a boundary layer located in the inner disk (Shakura \& Sunyaev 1973; Lynden-Bell \& Pringle 1974; Pringle \& Savonije 1979; Papaloizou \& Stanley 1986; Popham et al. 1993; Bisnovatyi-Kogan 1994; Popham \& Narayan 1995). This takes place via turbulent friction between differentially rotating layers in the disk. Thus X-ray emission would take place in this inner disk.

Inogamov \& Sunyaev (1999) recently proposed a radically different approach in which the adjustment in angular velocity takes place on the surface of the star, material meeting the star in the equatorial plane and flowing vertically on the stellar surface away from the plane and forming a spreading layer. Viscous interaction with the underlying surface takes place while the angular velocity of the flow material differs from that of the star, leading to heating and X-ray emission which rises to a peak at a particular arc distance $R \theta$ measured on the surface of the star from the equator. The emitted flux then falls rapidly with $R \theta$ as energy is radiated. Thus, for a given luminosity, there is a band on the surface of the star where the X-ray emission takes place. A system of equations was set up for the spreading layer considered essentially as a stellar atmosphere, consisting of hydrostatic equilibrium, radiative equilibrium, an equation of state containing gas and radiation pressure and the Stefan-Boltzmann law. The opacity was assumed to be dominated by Thomson scattering. Transformation and solution of these equations was carried out for a number of values of accretion rate, i.e. neutron star spreading layer luminosity. In particular, results were presented for the variation of X-ray flux $q$ defined as the energy emitted per unit area per second, with arc distance. Figure 3 reproduces the results of Inogamov \& Sunyaev for the distribution of X-ray flux with $R \theta$ for 4 values of the neutron star luminosity equal to $1 \%, 4 \%, 20 \%$ and $80 \%$ 


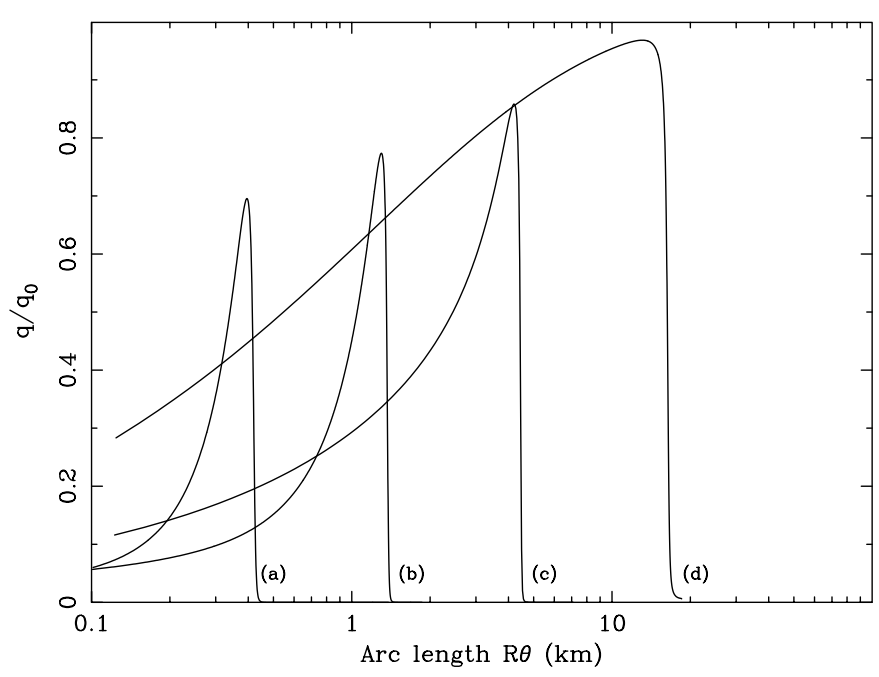

Fig. 3. Variation of X-ray flux $q$ emitted from the neutron star expressed as a fraction of the Eddington flux $q_{0}$ with arc length $R \theta$ for 4 cases in which the source luminosity is (a) $1 \%$, (b) $4 \%$, (c) $20 \%$ and (d) $80 \%$ of the Eddington limit.

of the Eddington limit $L_{\mathrm{Edd}}$. The flux is given relative to the Eddington flux $q_{0}$, where

$L_{\mathrm{Edd}}=4 \pi R^{2} q_{0}=1.26 \times 10^{38} \frac{M}{M_{\odot}} \operatorname{erg~s}^{-1}$,

and

$q_{0}=\frac{G M m_{\mathrm{p}} c}{R^{2} \sigma_{\mathrm{T}}}$

where $M$ is the mass of the neutron star, $m_{\mathrm{p}}$ is the proton mass and $\sigma_{\mathrm{T}}$ is the Thomson cross section. $L_{\mathrm{Edd}}$ is, of course, independent of the radius assumed for the neutron star. Figure 3 shows $q / q_{0}$ integrated over photon energy as a function of arc length assuming $q_{0}$ appropriate to a $1.4 M_{\odot}$ object so that $L_{\mathrm{Edd}}$ is $1.76 \times 10^{38} \mathrm{erg} \mathrm{s}^{-1}$. For the 4 cases, the neutron star luminosities are $1.76 \times 10^{36}, 7.04 \times 10^{36}, 3.52 \times 10^{37}$ and $1.67 \times 10^{38} \mathrm{erg} \mathrm{s}^{-1}$.

Clearly, at low luminosities the X-ray emission peaks in a narrow strip relatively close to the orbital plane, but extending down to $\theta=0$. At high luminosities the hot region expands to fill the star. Inogamov \& Sunyaev showed that the calculated fluxes were not sensitive to the magnitude of the viscosity parameter, as might be expected. X-ray observations are not capable, of course, of revealing the values $R \theta_{1}$ and $R \theta_{2}$ between which most of the $\mathrm{X}$-ray emission is concentrated assuming the model to be correct, but they do provide values of the emitting area from fitting a simple blackbody. From the measured luminosity and temperature, an area can be derived and compared with the emitting area of the neutron star in the Inogamov \& Sunyaev theory, as described in the next section.

\section{Results}

Figure 3 shows the variation of flux with polar angle $q(R \theta)$ expressed as a fraction of the Eddington flux $q_{0}$ in the theory of Inogamov \& Sunyaev which we wish to compare with the results of the ASCA survey of LMXB. Although Inogamov \& Sunyaev use an electron scattering dominated opacity for the
Table 1. Emitting region on the surface of the neutron star calculated using the theory of Inogamov \& Sunyaev (1999) expressed in terms of the range of $R \theta$ and also in terms of half-heights $h$ derived for these for 4 luminosities of the spreading layer $L_{\mathrm{SL}}$.

\begin{tabular}{lrrrc}
\hline \hline$L_{\mathrm{SL}} / L_{\mathrm{Edd}}$ & $\Delta R \theta$ & $\begin{array}{c}h \\
F W H M \\
\%\end{array}$ & $\mathrm{~km} \theta$ & $\begin{array}{c}h \\
I_{0.5}^{0.99} \\
\mathrm{~km}\end{array}$ \\
\hline 1 & 0.12 & 0.12 & 0.09 & 0.09 \\
4 & 0.44 & 0.44 & 0.32 & 0.32 \\
20 & 2.47 & 2.45 & 1.51 & 1.50 \\
80 & 15.81 & 11.62 & 9.14 & 8.28 \\
\hline
\end{tabular}

surface layers of the neutron star, they in fact, implement their model assuming simple blackbody emission. As discussed in Sect. 1, whether modification of the blackbody takes place depends on the electron density, and no evidence for modification in non-burst emission is known to exist. Thus we simply obtain the theoretical sizes of the hot regions on the neutron star from Fig. 3 and compare these with blackbody areas from the survey.

We adopt two ways of defining the emitting area from the theoretical variation $q(R \theta)$; comparison of these will indicate the uncertainty in the area. Firstly, we use the region on the neutron star corresponding to the $F W H M$ of the flux $q(R \theta)$, i.e. the range between points where $q=q_{\max } / 2$. Secondly, we used a definition based on the intensity $I \propto \int q\left(\theta^{\prime}\right) \cos \theta^{\prime} \mathrm{d} \theta^{\prime}$ integrated over the surface of the sphere allowing for the angular dependence of the area $2 \pi R^{2} \cos \theta \mathrm{d} \theta$. The intensity $I(\theta)$ increases asymptotically to a constant value corresponding to the maximum value of $R \theta$ at which there is $\mathrm{X}$-ray emission. The range of $R \theta$ was then obtained between $I=0.50 I_{\max }$ and $0.99 I_{\max }$. For each method, we calculated the half-height $h$ of an equatorial strip having the same area, from the range of $R \theta$ values using $h=R \sin \theta$ with $12 \mathrm{~km}$ for the radius of the neutron star as assumed by Inogamov \& Sunyaev. These data are shown in Table 1. The case of the highest luminosity point is interesting. The $F W H M$ in this case gives a value of $\Delta(R \theta)$ of $15.8 \mathrm{~km}$ which corresponds to $\Delta \theta=75.5^{\circ}$ if we assume $R=12 \mathrm{~km}$, and $90.5^{\circ}$ for $R=10 \mathrm{~km}$. Thus, in the latter case, the whole star is clearly emitting and the value of $h$ equal to $R \sin \theta$ becomes $10 \mathrm{~km}$. Alternatively, if we assume that $15.8 \mathrm{~km}$ is one quarter of the circumference of the star, a radius of $10.1 \mathrm{~km}$ for the neutron star is obtained.

In Fig. 4 we compare values of $h$ calculated in these two ways with $H\left(r_{10}\right)$, the height of the inner disk, as a function of total luminosity. This function was shown to agree well with the ASCA survey results by Church \& Bałucińska-Church (2001), and was calculated assuming a neutron star radius of $10 \mathrm{~km}$. Open squares show the $99 \%$ intensity points, and open circles show the $F W H M$ points. It can be seen that there is less than a factor of two difference between the theoretical points of Inogamov \& Sunyaev for the two methods of area calculation. However, the contribution of the neutron star blackbody is only part of the total luminosity in X-ray binaries and so the theoretical points should be corrected. The other major emission 


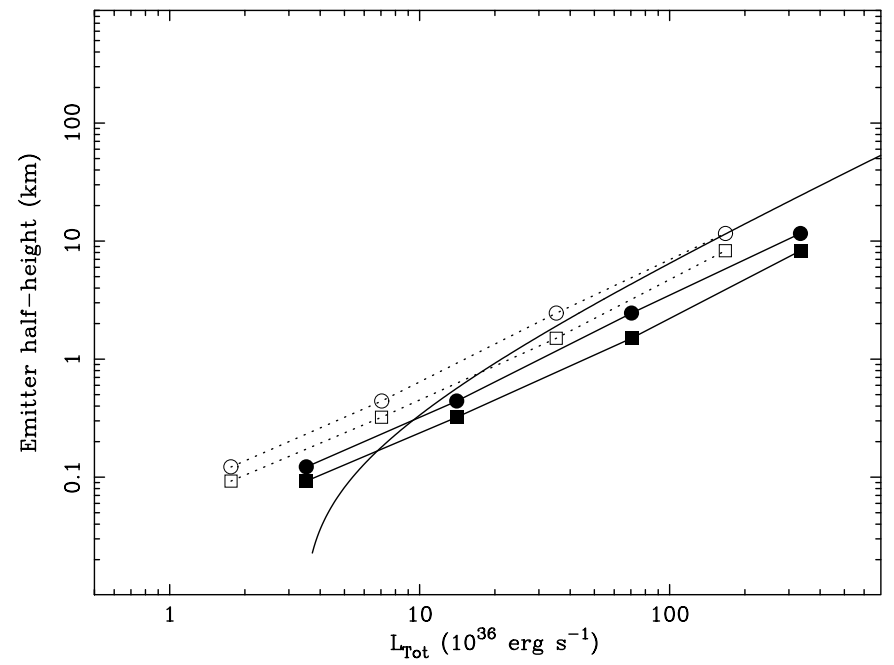

Fig. 4. Comparison of 4 theoretical points for the vertical extent of the emitting region on the neutron star with $H\left(r_{10}\right)$ (solid curve), the height of the disk where $p_{\mathrm{r}}=10 p_{\mathrm{g}}$. Open squares and open circles are uncorrected (see text); the squares show the height equivalent to $99 \%$ of intensity, the circles show the height equivalent to the FWHM of the flux distribution. Filled squares and filled circles are at corrected values of the total luminosity.

component is that arising originally as thermal emission in the accretion disk, which is then Comptonized in the ADC (e.g. Church 2001; Bałucińska-Church et al. 2001) making a dominant contribution in most LMXB. As Inogamov \& Sunyaev (1999) assumed the Newtonian approximation in which the energy available at the inner edge of the accretion disk is one half of the total energy, one possible method of correction would be to plot the points at $L_{\mathrm{Tot}}=2 \cdot L_{\mathrm{BB}}$, where $L_{\mathrm{BB}}$ is the luminosity of the spreading layer. The points corrected in this way are shown as filled circles and squares. The differing values of neutron star radius used by Inogamov \& Sunyaev $(12 \mathrm{~km})$ and in the calculation of $H\left(r_{10}\right)(10 \mathrm{~km})$ will have only $10 \%$ effect on the comparison.

In Fig. 5 we add the survey data points to the comparison. The theoretical points are shown as before, uncorrected and also corrected by a factor of two shift in $L_{\mathrm{Tot}}$. We now consider the extent of the required correction further. Inogamov \& Sunyaev (1999) assume that the spin of the neutron star is essentially zero, and Sibgatullin \& Sunyaev (1998) argue that in this case, $L_{\mathrm{BB}}=2.8 \cdot L_{\mathrm{Tot}}$. This implies that $L_{\mathrm{Tot}}=1.34 \cdot L_{\mathrm{BB}}$, so that we should correct the theoretical points in Figs. 3 and 4 by moving them to higher values of $L_{\mathrm{Tot}}$ by $34 \%$. However, this argument predicts that the spectra of LMXB in general would be strongly dominated by the thermal emission of the star, whereas, in fact, in many sources the Comptonized emission strongly dominates, and work on the dipping sources has proven that this originates in an extended ADC. It has been suggested, for example by Inogamov \& Sunyaev (1999) that modification of the neutron star emission in the stellar atmosphere will take place; however this will not lead to a power law spectrum as observed, but to a spectrum very similar to a simple blackbody (Madej 1991) and the observed dominance of Comptonized emission in the spectrum could only be avoided

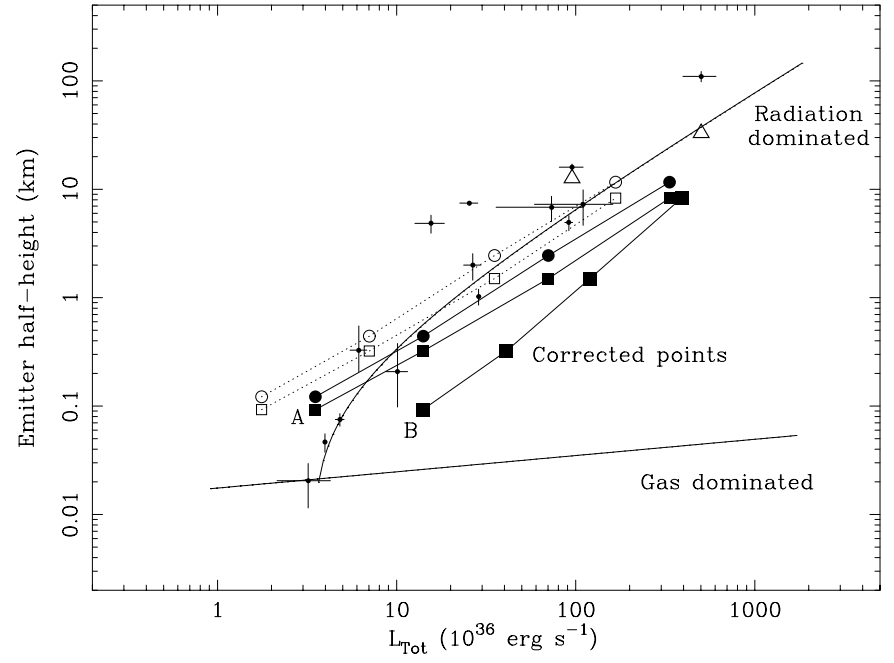

Fig. 5. Same as Fig. 4 with data points from the LMXB survey added for luminosities in the band $1-30 \mathrm{keV}$. The set of points labelled $\mathrm{A}$ are corrected as in Fig. 4. The points labelled B are corrected in luminosity based on observational information from the LMXB survey. Also shown is the variation of the thin disk half-height calculated close to the stellar surface (at $1.001 R$ ) with $L$. The theoretical height of the radiatively-supported disk (curved line) approaches the thin disk line at low $L$ where radiative support fails.

by assuming that most LMXB have a neutron star spinning sufficiently fast that the emission due to viscous dissipation in the surface is markedly reduced. As an alternative to seeking a theoretical relation between $L_{\mathrm{Tot}}$ and $L_{\mathrm{BB}}$, we can use an approximate observational relationship provided by the ASCA survey. Figure 1 shows that there is not a simple relationship between $L_{\mathrm{BB}}$ and $L_{\mathrm{Tot}}$, but rather that the blackbody luminosities occupy a broad band of the two-dimensional space. To make an approximate correction, we use the centre of this band. This requires a substantial movement of the theoretical points in Fig. 5 to higher values of $L_{\text {Tot }}$ as shown by the filled squares labelled B.

However, we can avoid the difficulty of the correction by comparing the theoretical points with the survey data replotted in terms of $L_{\mathrm{BB}}$ instead of $L_{\mathrm{T}}$ as shown in Fig. 6. A curve showing the theoretical height of the accretion disk is not shown as this is a function of $L_{\mathrm{Tot}}$. It can be seen that the survey points tend to converge with the theory at the lowest luminosity, but are substantially larger than the theoretical points at higher luminosities.

\section{Discussion}

The main result of the ASCA LMXB spectral survey was the agreement of $h=H$, for sources in the quiescent state (i.e. non-flaring). However, it is not clear which of two explanations of this is correct: that of radial flow between inner disk edge and star, in which the height of the inner radiatively supported disk directly determines $h$, or the theory of Inogamov \& Sunyaev (1999) in which spreading of material on the neutron star surface to height $h$ depends on the mass accretion rate of which $H$ is a measure. In the case of the first mechanism, the 


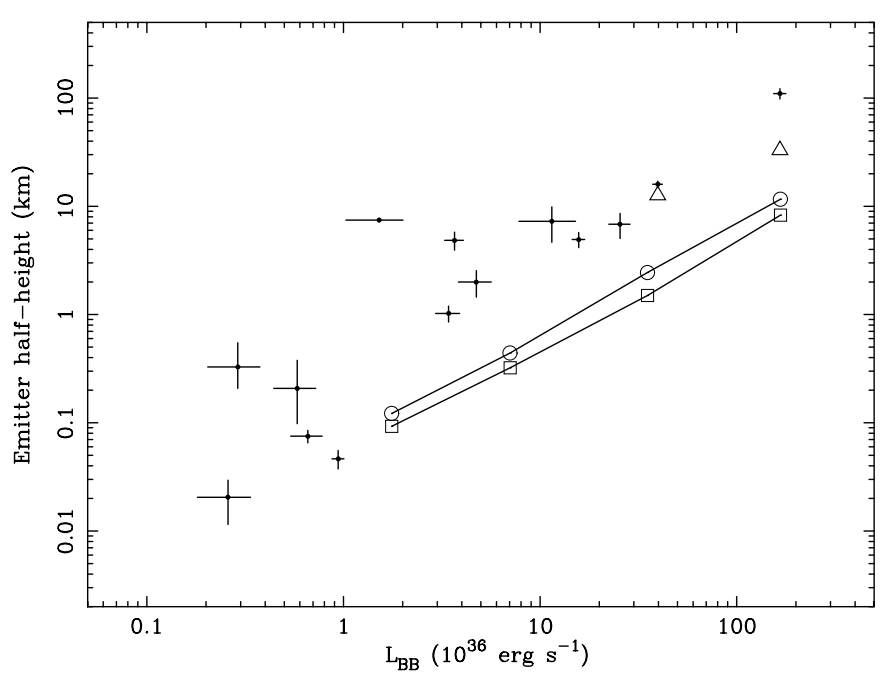

Fig. 6. The 4 theoretical points plotted as a function of blackbody luminosity compared with data from the survey in the band $1-30 \mathrm{keV}$.

inner disk may have the steep radial profile implied by standard theory close to the neutron star, but radial flow takes place across the gap between inner disk and star thus defining the emitting region on the star of similar height to the highest point of the inner disk. Although there has been extensive theoretical investigation of advective flow in black hole systems (e.g. Abramowicz et al. 1996), very little work has been carried out for neutron star binaries, and it is not clear whether the sonic point lies within the stellar surface or not. However, Popham \& Sunyaev (2001) show that the radial velocity increases by two orders of magnitude within the disk. Two- or three-dimensional modelling would be required to decide whether radial flow across the gap can take place. A possible objection to the first mechanism is that the radiatively supported inner disk may not be stable, i.e. implying that it cannot exist. The stability of the inner disk in a Galactic black hole system GRS 1915+109 has been considered theoretically by Janiuk et al. (2000) who showed that oscillations are expected leading to periodic variations in the X-ray luminosity of the system similar to those observed. However, it was not found that this leads to major disruption of the disk. Since the timescale for variability depends on mass (Czerny, priv. comm.), we have applied this model to LMXB by scaling the stellar masses, and find a timescale of $\sim 30 \mathrm{~s}$. Variability on this timescale is not obviously observed, and would not in any case prove that major disruption of the disk takes place.

In the present work, we have compared the survey results with the theory of Inogamov \& Sunyaev (1999) in two ways. Firstly, we made an approximate comparison as shown in Figs. 4 and 5. When a more appropriate comparison is made by comparing with $L_{\mathrm{BB}}$, not $L_{\mathrm{Tot}}$, as shown in Fig. 6, the survey results and theory converge at the lowest luminosities, but at higher luminosities, the data, i.e. the emitting areas, exceed the theory by a factor that we estimate as between four and eight. However, the methods used of obtaining the emitting area from theory underestimates the area, for example by using the $F W H M$, by up to a factor of two. It consequently appears that the data exceed the theory by a factor of two to four. Thus at low luminosities, the emitting area on the neutron star may be determined by accretion spreading, although even here radial flow would be an alternative explanation. At higher luminosities, the area exceeds the spreading theory implying that the area is determined by radial flow which leads to the observed agreement between $h$ and $H$ and which enhances the contribution due to spreading. Although there is no observational evidence for any modification of the neutron star blackbody spectrum in the non-burst emission of a LMXB, this would lead to an underestimation of the emitting area, and the disparity between the survey results and spreading theory would be somewhat increased.

Extension of the present accretion flow spreading theory which is essentially one-dimensional, to a two-dimensional form would be desirable. Further analysis of LMXB spectra is clearly needed to increase the size of the sample and to make more use of BeppoSAX data to constrain spectral fitting parameters better. However, the present results indicate that while the neutron star accretion flow spreading theory of Inogamov \& Sunyaev may set a base level to the neutron star blackbody emission, as observed in the faintest sources, it underestimates the observed blackbody luminosity in brighter sources. This implies that radial flow dominates leading to the agreement between $h$ and $H$.

\section{References}

Abramowicz, M. A., Chen, X.-M., Granath, M., \& Lasota, J.-P. 1996, ApJ, 471, 762

Bałucińska-Church, M., Church, M. J., Oosterbroek, T., et al. 1999, A\&A, 349, 495

Bałucinska-Church, M., Humphrey, P. J., Church, M. J., \& Parmar, A. N. 2000, A\&A, 360, 583

Bałucinska-Church, M., Barnard, R., Church, M. J., \& Smale, A. P. 2001, A\&A, 378, 847

Bisnovatyi-Kogan, G. S. 1994, MNRAS, 269, 557

Church, M. J. 2001, Proc of 33rd Scientific Assembly of COSPAR, Warsaw, July 2000, Adv. Space Res., 28, 323

Church, M. J., \& Bałucińska-Church, M. 2001, A\&A, 369, 915

Church, M. J., Dotani, T., Bałucińska-Church, M., et al. 1997, ApJ, 491, 388

Church, M. J., Parmar, A. N., Bałucińska-Church, M., et al. 1998, A\&A, 338, 556

Ebisuzaki, T. 1987, PASJ, 39, 287

Ebisuzaki, T., Hanawa, T., \& Sugimoto, D. 1984, PASJ, 36, 551

Fujimoto, M. Y., Hanawa, S., \& Miyaji, S. 1981, ApJ, 246, 267

Czerny, B., \& Elvis, M. 1987, ApJ, 321, 305

Guainazzi, M., Parmar, A. N., Segreto, A., et al. 1998, A\&A, 339, 802

Inogamov, N. A., \& Sunyaev, R. A. 1999, Astron. Lett., 25, 269

Inoue, H., Waki, I., Koyama, K., et al. 1984, PASJ, 36, 831

Janiuk, A., Czerny, B., \& Siemiginowska, A. 2000, ApJ, 542, L33

Kuulkers, E., Homan, J., van der Klis, M., Lewin, W. H. G., \& Mendez, M. 2002, A\&A, 382, 947

London, R. A., Taam, R. E., \& Howard, W. M. 1984, ApJ, 287, L27

London, R. A., Taam, R. E., \& Howard, W. M. 1986, ApJ, 306, 170

Lynden-Bell, D., \& Pringle, J. E. 1974, MNRAS, 168, 603

Madej, J. 1991, ApJ, 376, 161

Madej, J., \& Różańska, A. 2000, A\&A, 356, 654 
Mitsuda, K., Inoue, H., Nakamura, N., \& Tanaka, Y. 1989, PASJ, Sibgatullin, N. R., \& Sunyaev, R. A. 1998, Astron. Lett., 24,774 41,97

Shakura, N. I., \& Sunyaev, R. A. 1973, A\&A, 24, 337

Oosterbroek, T., Parmar, A. N., Sidoli, L., in't Zand, J. J. M., \& Heise, J. 2001, A\&A, 376, 532

Paczyński, B. 1983, ApJ, 267, 315

Shakura, N. I \& Sunyaev, R A 1976, MNRAS, 175, 613

Sidoli, L., Parmar, A. N., Oosterbroek, T., et al. 2001, A\&A, 368, 451

Papaloizou, J. C. B., \& Stanley, G. Q. G. 1986, MNRAS, 220, 593

Pringle, J. E., \& Savonije, G. J. 1979, MNRAS, 187, 777

Popham, R., \& Narayan, R. 1995, ApJ, 442, 337

Popham, R., \& Sunyaev, R. A. 2001, ApJ, 547, 355

Smale, A. P., Church, M. J., \& Bałucińska-Church, M. 2001, ApJ, 550, 962

Sztajno, M., van Paradijs, J., Lewin, W. H. G., et al. 1985, ApJ, 299, 487

Popham, R., Narayan, R., Hartmann, L., \& Kenyon, S. 1993, ApJ, 415, Sztajno, M., van Paradijs, J., Lewin, W. H. G., et al. 1986, MNRAS, L127 222,490

Rutledge, R. E., Bildsten, L., Brown, E. F., Pavlov, G. G., \& Zavlin, V. E. 1999, ApJ, 514, 945

(1994, ApJ, 434, 313

White, N. E., Stella, L., \& Parmar, A. N. 1988, ApJ, 324, 363 